\title{
Intelligent Micro Grid Research at BCIT
}

\author{
Hassan Farhangi, PhD, PEng, SM-IEEE \\ Director, Group for Advanced Information Technology \\ British Columbia Institute of Technology, Burnaby, BC \\ hassan_farhangi@bcit.ca
}

\begin{abstract}
This paper describes a major research initiative by British Columbia Institute of Technology for the construction of an Intelligent Micro Grid on its campus in Burnaby, BC, Canada.

Keywords-Intelligent Grid; Utility; Renewable Energy; MicroGrid; Intelligent Agents; Intelligent Appliance; Electricity Grid; Smart Grid; Distributed Generation; Co-Generation; Photovoltaic;
\end{abstract}

\section{INTRODUCTION}

Utility companies across the world have recognized the need for a major technological overhaul of their assets. As the backbone of the power industry, the electricity grid is now the focus of assorted technological innovations. Utilities need to introduce distributed intelligence into their existing infrastructure to make them more reliable, efficient and capable of exploiting and integrating diverse sources of energy, including alternative energy sources [1].

The Intelligent Grid needs to be designed with efficiency in mind, providing for distributed control and monitoring using smart components. This will allow optimal use of expensive assets through demand response, fair energy pricing and customer education.

The Intelligent Grid (also known as the "Smart Grid") includes the infrastructure and the technologies required to facilitate distributed generation and co-generation of energy. It provides for the integration of alternative sources of energy and management of system's emissions, and carbon footprint.

In addition to enabling utilities to bring renewable sources of energy into the mainstream, the Intelligent Grid's most immediate impact is to allow efficient and optimal use of utilities' existing assets through demand response, peak shaving and service quality control.

However, despite the urgent need to materialize the Intelligent Grid, certain impediments have slowed down the implementation process. Most notable among these are the absence of standards, regulatory challenges, funding constraints, etc. While such issues need to be dealt with in the context of utility providers' overall business framework, help is needed to address other obstacles which are highly technical in nature [2].

The problem that most utility providers face is not the absence of concepts, ideas and technologies. On the contrary, a multitude of disparate technologies have been developed by the industry (e.g. communication protocols, computing engines, smart sensors, intelligent algorithms, control models, etc), which can potentially address utility applications and resolve issues within the Intelligent Grid [3].

Nevertheless, such technologies have not yet been proven in the context of utility providers' desired specifications, configurations and architecture. And given the huge responsibility which utilities have in operating and maintaining such critical infrastructure, they would find it hard to venture into new territories, new technologies and new solutions. This critical issue, which is slowing down the pace at which technological innovations can find their way from labs to the field, is addressed by BCIT's Intelligent Micro Grid Initiative.

\section{BCIT'S INTELLIGENT MICRO GRID INITIATIVE}

\section{A. Overview}

The British Columbia Institute of Technology (BCIT), is in the process of designing and developing a scaled-down version of the Intelligent Grid, i.e. an Intelligent Micro Grid to enable utility companies, technology providers and researchers to work together to develop and facilitate the commercialization of architectures, protocols, configurations and models of the evolving Intelligent Grid with the intention of charting a "path from lab to field" for innovative and costeffective technologies and solutions for North America's evolving Smart Electricity Grid [4].

BCIT's Intelligent Micro Grid is an RD\&D (Research, Development and Demonstration) platform where existing and future technologies in telecommunication, smart metering, cogeneration and intelligent appliances are employed to develop and qualify the most robust, cost-effective and scalable solutions required to facilitate and nurture the evolution and the emergence of the Smart Grid in one form or another.

BCIT has assembled a consortium of private industry partners, referenced here as BMPIC (BCIT Micro grid Private Industry Consortium) to help with the design and implementation of BCIT's Intelligent Micro Grid. BMPIC is composed of local and international technology companies which are at the forefront of technology developments in this field. BMPIC covers a wide array of hardware, software and system technologies required for the realization of Intelligent Grid $\uparrow$. 
The validation and qualification of architectures, models and protocols developed for the Intelligent Micro Grid is guided, supervised \& scrutinized by the partnering utility companies as end-customers of the developed technologies and solutions. BCIT's Intelligent Micro-Grid is implemented at BCIT's Main Campus in Burnaby.

BCIT's Intelligent Micro-Grid enables high-tech companies, end customers and researchers to work together to develop and qualify various system architectures, configurations, interface protocols and grid designs to meet national and global priorities for co-generation, efficient transmission and distribution of electricity, load control, demand response, advanced metering and integration of clean energy sources into the existing and future grids.

\section{B. Key Deliverables}

Funded in part by BC Government's Innovative Clean Energy Fund (ICE Fund), and in-kind contributions and support by BMPIC members, the key deliverables of this project over its three consecutive phases are the following:

1. Construction of an RD\&D platform to verify:

a. provisioning methods for smart termination points (meters, data aggregators, appliances, sensors, controls, etc)

b. integration solutions for alternative sources of energy (co-generation using wind, solar, thermal, bio, etc) with the electricity grid

c. innovative network architecture and topologies for smart grid

2. Operational analysis and qualification of grid's:

a. resilience, reliability, security and scalability

b. data collection and distributed command and control methods

c. emission standards for wood waste boilers and methods for achieving such standards with optimum efficiencies

3. Development and qualification of Intelligent Agents, protocols and models to ensure:

a. efficient interface with Utility back-office tools (billing, load management, service provisioning, outage restoration, etc)

b. seamless end-to-end deployment, operation and maintenance of essential services

C. easy and intuitive human interface for operators and customers

\section{MAJOR COMPONENTS OF BCIT'S MICRO-GRID}

The Intelligent Grid achieves operational efficiency thru distributed control and monitoring. This allows for optimal use of assets through demand response, fair energy pricing and customer education.
The Intelligent Grid (also known as the "Smart Grid") includes the infrastructure and the technologies required to allow distributed generation, storage and consumption of energy. It provides for efficient integration of alternative sources of energy and interactive and real-time management of system's resources and assets.

In addition to enabling utilities to bring renewable sources of energy into the mainstream, the Intelligent Grid's most immediate impact is to allow efficient and optimal use of utilities' existing assets through customer education, revenueleakage-prevention, demand response and peak shaving.

In order to closely replicate the operational environment within which such concepts need to be developed and qualified, BCIT's Intelligent Micro-Grid strives to incorporate all but one of the essential building blocks of a typical manifestation of an Intelligent Grid. As such, a full spectrum of load profiles, various co-gen plants, distribution, control and monitoring systems are incorporated into BCIT's RD\&D setup.

To counter the intermittent availability of renewable energy sources, energy storage components such as fuel cells are required. At the time of writing of this paper, such components have not yet made their way into BCIT's Micro-Grid infrastructure. Plans are being drawn to incorporate storage elements into the components mix in the not too distant future.

\section{A. Physical Setup}

BCIT's Burnaby Campus is one of the largest postsecondary campuses in the Greater Vancouver Regional Area. It resembles a small neighborhood, containing over 50 buildings. The campus houses classrooms, administration buildings, workshops, food outlets, student services and dormitories.

The sheer size and the diversity of activities requires BCIT's Burnaby Campus to cater to a variety of different electricity consumption profiles ranging from heavy industrial machinery for instructional purposes in the workshops, to office type consumption in classrooms along with a residential-type profile in dormitories.

In addition to such diverse usage patterns, the campus includes co-generation power plants. There is an existing photovoltaic tower, capable of generating up to $8 \mathrm{KW}$ of electricity, as well as three smaller PV systems elsewhere on the campus with a total generation capacity of around $14 \mathrm{KW}$. Work is also underway to equip the campus with a power boiler and a steam turbine-generator.

Moreover, as part of the future expansion of this initiative, BCIT is planning to add to its Intelligent Micro-Grid two additional co-generation sources at the BCIT Burnaby Campus. 
The first of the future campus-based co-generators is an industrial-scale micro-wind turbine and generator which is based on a BCIT-developed wind-accelerator technology designed for areas where the wind intensity is normally insufficient for power generation.

BCIT's proven and patent-pending technology involves the use of a specially designed concentrator to accelerate and amplify the velocity of the wind $\dagger \uparrow$.

The second campus-based co-generation source would involve an additional boiler fueled by wood-waste. This will be used to develop and verify efficient and environmentally friendly ways of producing energy from beetle-infested timber, a significant ecological disaster in British Columbia.

With its own electricity feeds from power utility and its own local substations and distribution infrastructure, as well as its own co-generation capabilities, the BCIT Burnaby Campus can be technically regarded as a true Micro-Grid.

Moreover, the campus houses a state-of-the-art multi-milliondollar Network Engineering Lab, (also known as the Internet Engineering Lab, or "IEL") funded jointly by the government and the industry.

The IEL contains network hardware and test equipment including network routers and switches, traffic generators, impairment emulators and network analysis tools capable of emulating and testing both small and large-scale network configurations. The architecture of the IEL allows for a range of traffic levels and interconnection types so that different grid topologies can be experimented with in a realistic yet totally controlled environment.

To turn BCIT's campus into an Intelligent Micro-Grid, a network of smart terminations and control components is being created. Buildings are equipped with smart meters, appliances fitted with smart controls. All of these components are fitted with communication modules to enable them to communicate with data aggregation units in substations.

Furthermore, intelligent agents are being developed and embedded in all critical interface points within the system to gather, monitor and analyze the relevant data streams required to make global and/or local operational decisions to meet systems' service requirements and objectives. Networking of these agents is achieved through different network topology, architecture and protocols to be implemented in BCIT's Networking Lab (IEL).

In addition to the termination points, localized co-gen plants (solar, wind and thermal) are integrated into the Micro-Grid to allow for the development of command and control models for distributed power co-generation which is required for the integration of such alternative energy sources into the future Intelligent Grid.

\section{B. Main Topology}

As shown in the two Figures below, BCIT's Intelligent Micro-Grid is an integration of four entities; co-gen plants, campus loads, meshed network and core intelligence.

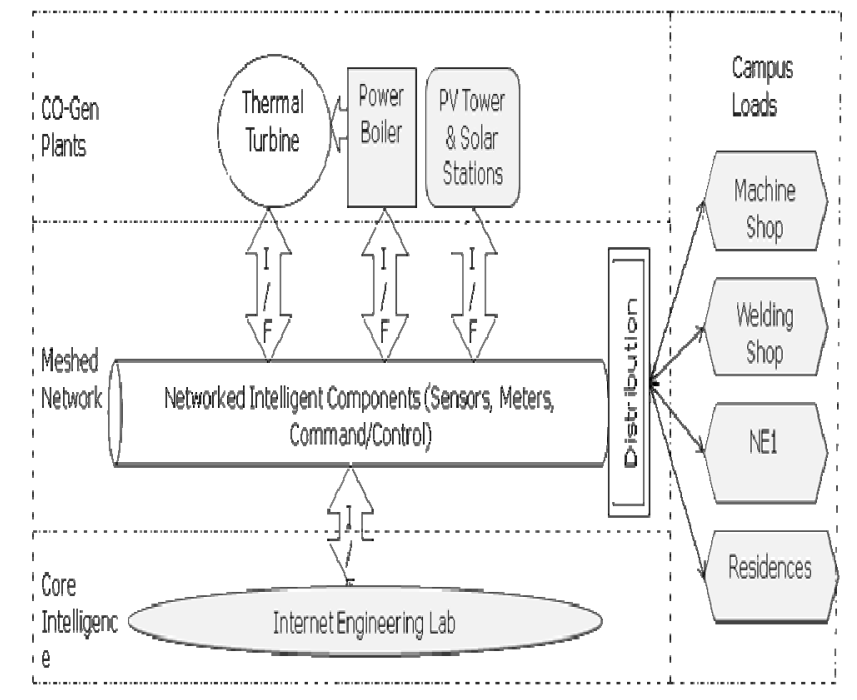

Note: Components denoted in grey are avisilable on the campus. Componenis denoted in white need to be acquired.

Figure 1a: Main components of BCIT's Micro Grid

Figure 1a depicts BCIT's Intelligent Micro Grid's components. However, as mentioned earlier, in the future two additional sources of co-generation from wind and bio-mass are planned for the project.

The extended system is depicted in Figure $1 b$.

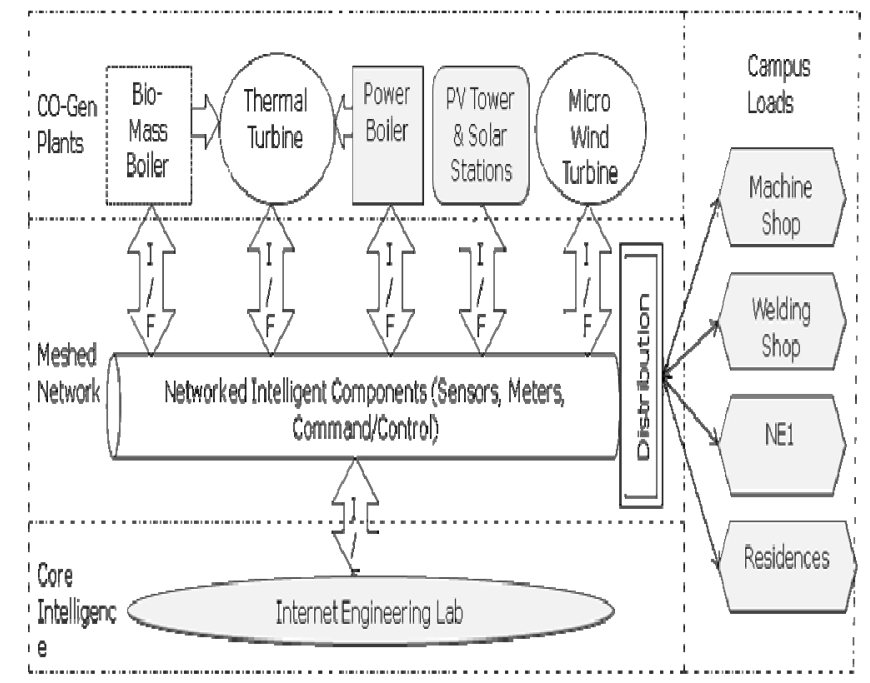

Note: Components denoted In grey are gyaliable on the campus Components denoted in whtte need to be acoulred,

Figure 1b: Extended components of BCIT's Micro Grid 


\section{Co-Generation Plants}

\section{Boilers}

A key component of the Intelligent Micro Grid project is BCIT's new state-of-the-art boiler facility and steam turbine co-gen plant. It completes the micro-grid environment by enabling smart sensors, which monitor the load demand, to bring the co-gen plants online to supply power to the BCIT Micro-Grid during peak consumption periods. The addition of a Bio-Mass boiler would enable BCIT to contribute to the practical implementation of solutions for a major issue - the use of beetle-infested wood for the production of electricity.

In fact, BCIT's Power Boiler is a_state-of-art environmentally friendly boiler which will meet the new GVRD's (Greater Vancouver Regional District) emission standards for all new boilers. It will provide a benchmark for achievable boiler standards and could potentially be used in the development and evaluation of proposed new standards.

Moreover, BCIT's Industrial Process Facilities is equipped with a boiler which will power the existing BCIT pulp-making installation and food processing lab. This may be used to study load shifting to and from the co-gen facility and used to simulate such processes common in industrial installations such as pulp mills.

Furthermore, smart sensors and control components are integrated into a steam turbine driving a generator implementing a sophisticated control system integrated with the boiler's burner management system for boiler control. This facility will also contain the necessary storage and switching gear required for the co-gen plant. The facility will be approximately 1,400 square feet.

Bio-mass boiler is designed to allow the use of fuel preprocessing methods untried in the industry. It is anticipated that the volume of beetle-infested wood that can be converted to electricity will be so significant that it is imperative to investigate a variety of efficient and environmentally friendly methods of producing electricity from this resource. Thus a bio-mass boiler similar to a hog fuel boiler (commonly used in the forest products industry) will be installed at the BCIT Campus

\section{Photovoltaic stations}

The BCIT Burnaby Campus is already equipped with four solar photovoltaic co-gen systems. An 8-kW grid-tied Photovoltaic (PV) tower is located at the east side of the campus (in front of Building SE1). This tower has four sides, each with 2-kW PV capacity. Inverter and monitoring systems are installed inside the tower. Interfaces to monitor the energy production are available as well. Figure 2 shows the tower and an interface screen.

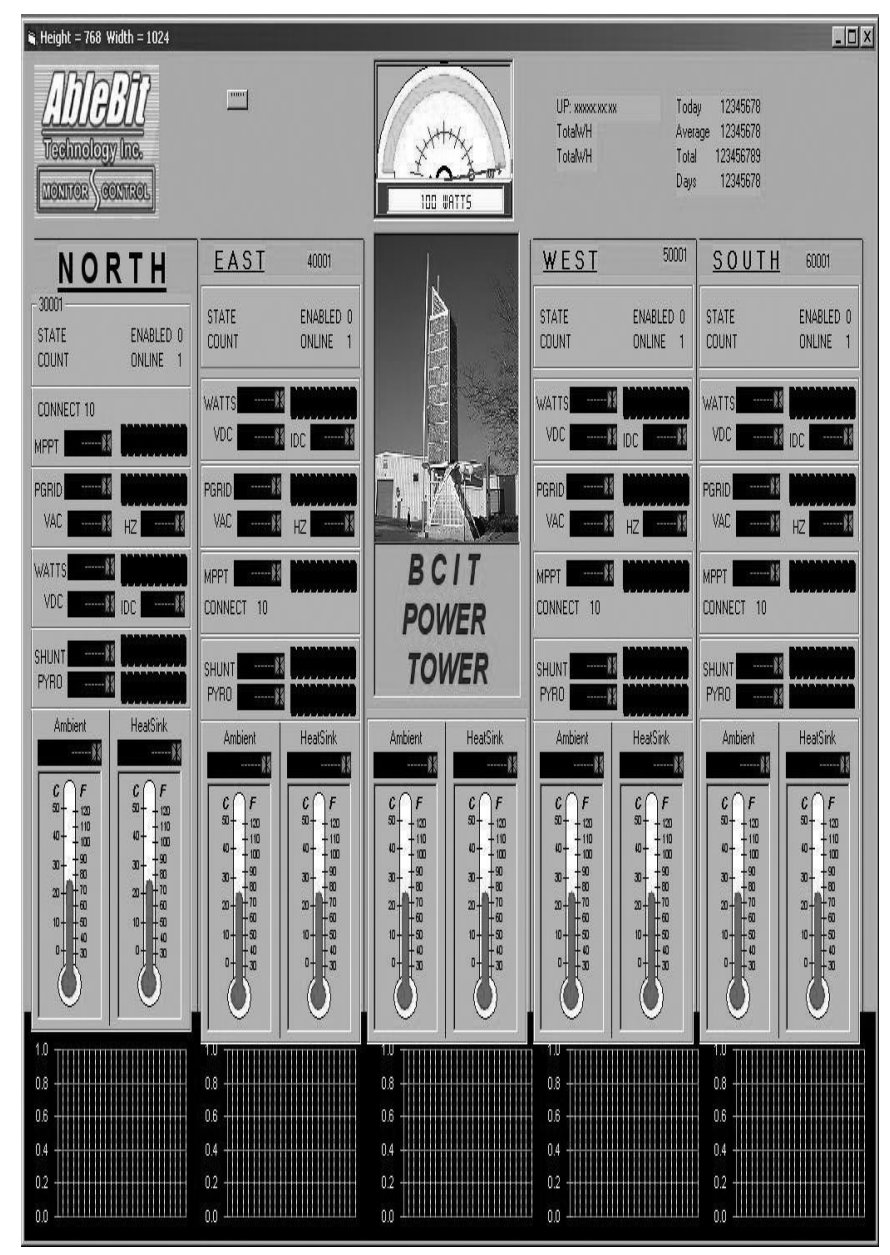

Figure 2: PV Tower \& Interface Screen

A 2-kW grid-tied PV system is installed on the east roof side of the AFRESH home, located at the north side of the campus (next to Building NE3). It generates electricity for the AFRESH building.

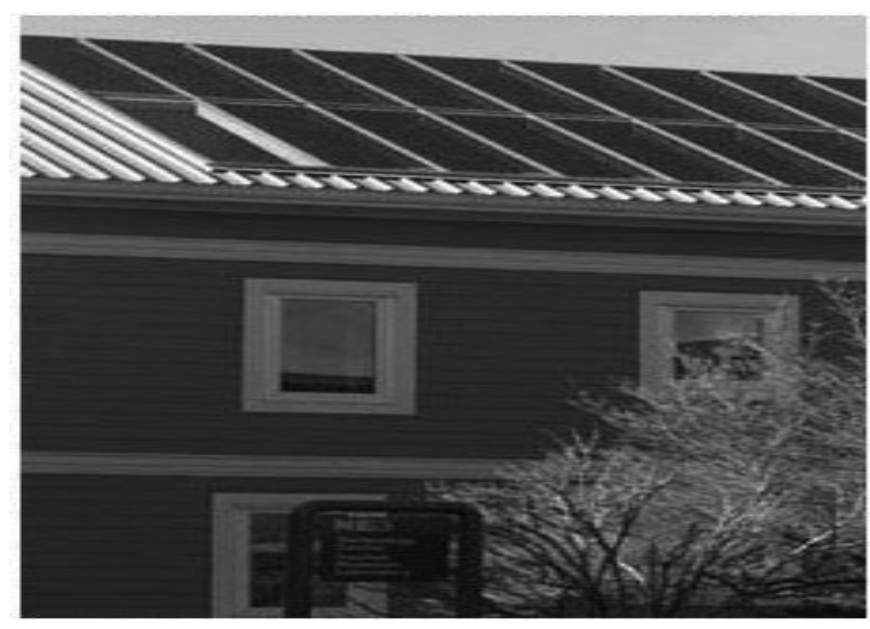

Figure 3: AFRESH PV System 
Two $1.4 \mathrm{~kW}$ grid-tied PV systems, as a curtain-wall, are installed on the facade of Technology Place, a researchpark/incubator building, that is located on the BCIT Campus. The system is shown in Figure 4.

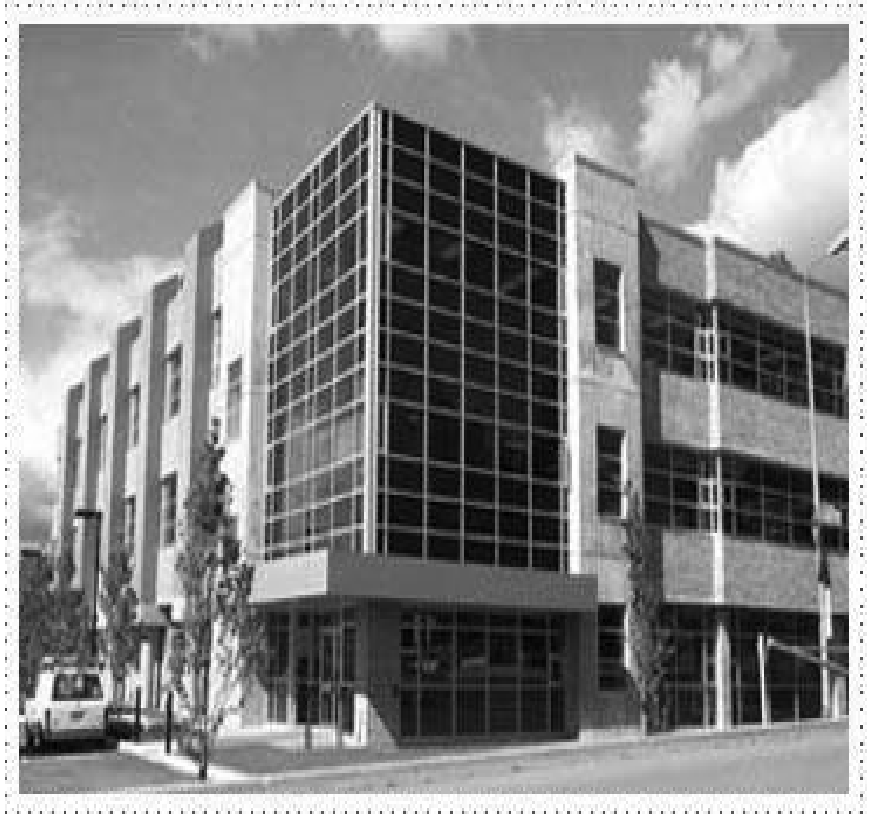

Figure 4: Technology Place PV System

The fourth photovoltaic system is a $1-\mathrm{kW}$ grid-tied PV system installed on a building in the northeast quadrant of BCIT's main campus. It is shown in Figure 5.

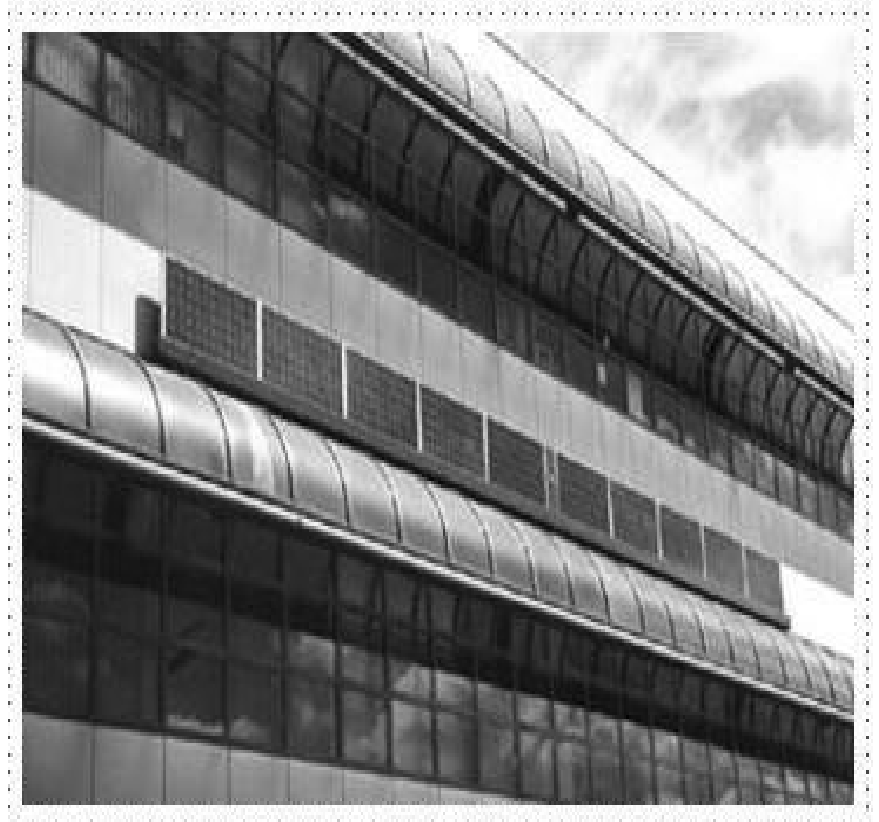

Figure 5: Technology Center PV System
3. Micro-Wind Turbine

A wind power plant will be considered as an extension to the initiative. It would be an industrial scale version of the Wind Powered Deflector (WPD), a small wind technology invented and developed at BCIT [5]. WPD technology enhances the power density available to a wind turbine by channeling air flow through an innovatively designed concentrator.

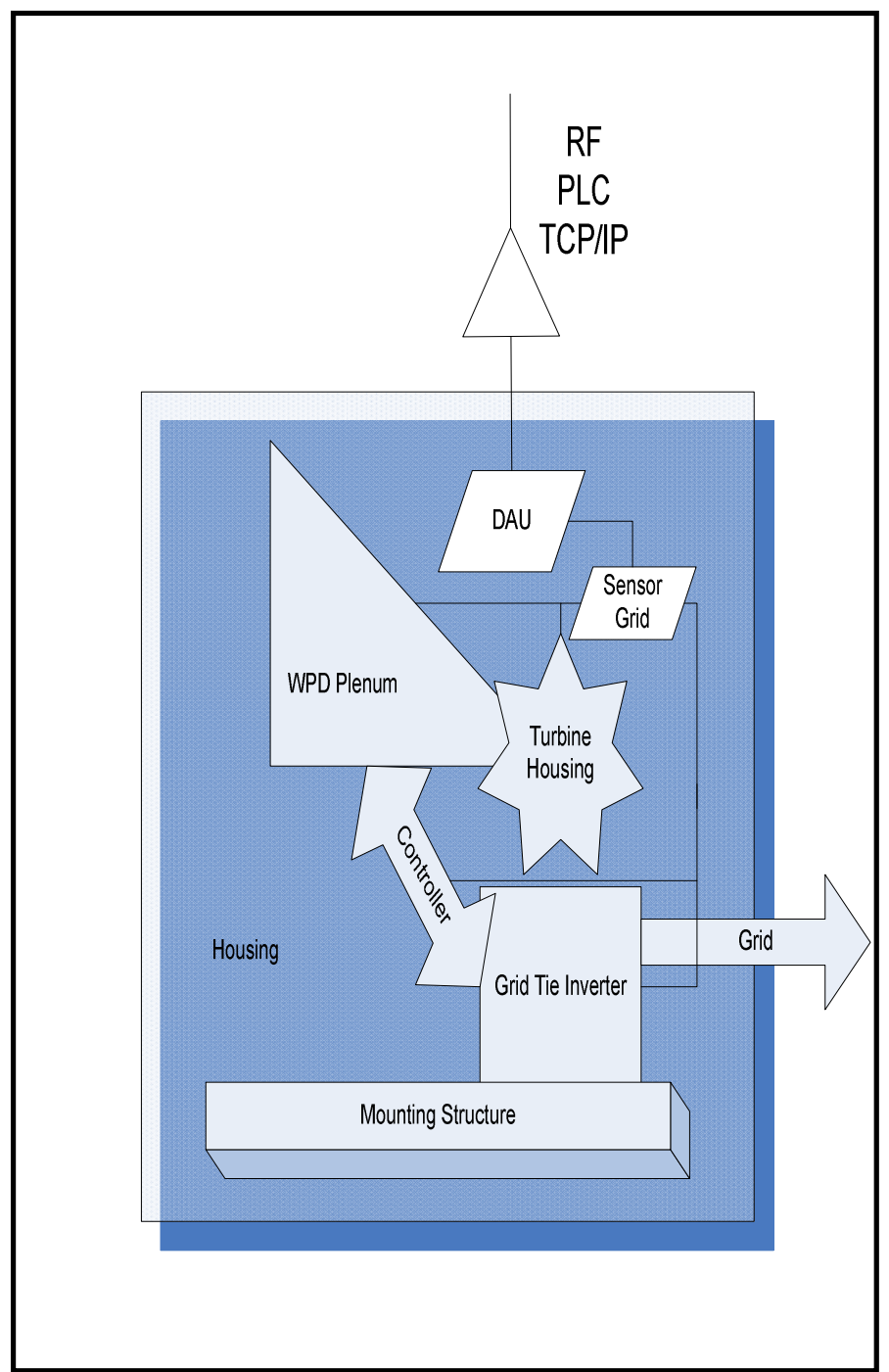

Figure 6: Wind Power Generator Block Diagram

Extensive experimental results using a model-scale of this technology have shown that WPD increases wind velocity by a factor of 3.7. This is of particular value to generating wind energy in urban areas where wind speeds are typically less than $4 \mathrm{~m} / \mathrm{s}$ (the minimum speed needed to provide sufficient kinetic energy to generate power by conventional turbines). Thus BCIT's WPD technology is seen as an innovative way of 
generating wind power in urban areas when augmented to a conventional small wind turbine $\dagger \dagger$.

\section{Campus Loads}

Four different buildings, with a variety of consumption profiles, are equipped with smart terminations. (1) The BCIT Machine Shop houses large $\mathrm{CNC}$ machines and heavy equipment mainly used for instructional purposes; (2) The BCIT Welding Shop contains numerous transformers and welding equipment, used for training; (3) Building NE1 contains several circuits supplying classrooms, cafeterias, offices, etc. and (4) Residences that are BCIT student dormitories.

These four buildings provide a rich set of service profiles prevalent in most urban settings. We are particularly interested in the power usage pattern, frequency, types of loads and applications which these buildings provide.

\section{E. Meshed Network}

The BCIT Intelligent Micro Grid Network integrates the following 7 components:

1. Smart Meters, capable of measuring multitudes of consumption parameters (e.g. active power, reactive power, voltage, current, demand, etc) with acceptable precision and accuracy. Smart Meters should be tamper-proof and capable of storing the required data for a number of billing cycles.

2. In-Home-Units, capable of measuring consumption profiles and displaying that in an easy-to-understand form for end-customers.

3. Communication Modules, enabling each meter to communicate with its adjacent meters, or with mobile/stationary data aggregators. The communication channel has to be extremely robust, secure and low-cost.

4. Access and Networking Middleware, to allow the setup and management of a secure meshed network comprising smart components in the same neighborhood, together with their assigned data aggregator.

5. Data Aggregator Unit (DAU), capable of exerting command and control over a meshed network of slave components. The Aggregator is capable of accessing each and every node in its assigned network.

6. Smart Appliances Control, capable of exerting command and control over Smart Appliances to adjust/control their performance and service level based on user and/or utility requirements.

7. Intelligent Agents, to allow authentication and handshake with each node in the network. It is capable of identifying each termination, querying them, exchanging data and command with them and managing the collected data for scheduled and/or on-demand transfer to Utility Servers.

The BCIT Micro-Grid will be rolled-out using different communication technologies such as RF, PLC, etc.

We shall make sure that the Micro-Grid is able to utilize and work with many different technologies which could become available in the future. The architecture shall be open and non-vendor specific.

\section{CORE INTELLIGENCE}

BCIT's Internet Engineering Lab (IEL) enables Data Aggregators to be networked with the utility control centers in a variety of different configurations, network topology and architectures. The WAN (Wide Area Network) will be emulated using BCIT's Internet Engineering Lab's routers, switches, firewalls and servers.

IEL's Servers shall be equipped with a selection of back-office utility tools to communicate with data aggregators, exchange command/control data and act as an interface between Intelligent Grid and Utilities' back-office software tools such as billing and accounting.

IEL will be collecting instantaneous and cumulative consumption data, alarms, power-quality and usage patterns from across the campus.

The system will be designed in such a way as to:

1. Ensure the integrity of time-sensitive data (event based and/or schedule based)

2. Ensure the reliability and security of communication channels

3. Parse data and make sense of what is important (which needs immediate attention) and what is not (which will be used for statistical reporting and archiving)

4. Mirror critical databases of substations

5. Present data in a useful and visual way (graphical outputs, interactive, query-based)

6. Develop predictive models of events and real-time responses

7. Manage distributed control to prevent cascading failures, or for the graceful degradation of user services based on service priorities

8. Real-time wide-area control to manage generation and prevent over-provisioning

9. Have context-dependent models and control of components to achieve robustness, fault-tolerance, or graceful performance degradation

10. Verify and qualify large-scale distributed real-time embedded systems

11. Develop support for integration and control of alternative and co-generation plants 
To achieve each of the above objectives, we need to work with our partners to arrive at the most suitable system architecture and topology. In doing so, we will be guided by the requirements and constraints specified by our end-customers.

\section{CONCLUSIONS}

BCIT has embarked on an exciting research initiative at its Burnaby campus, in Burnaby, BC. Inspired by $\mathrm{BC}$ Government's recently announced Green Energy Plan, BCIT is in the process of designing and constructing BC's (and we believe Canada's) very first Intelligent Power Micro Grid to enable Utility companies and researchers to work together to develop architectures, protocols, configurations and models of the evolving Intelligent Grid with the view to charting a "path from lab to field" for innovative and cost-effective technologies and solutions for the evolving Smart Electricity Grid.

The expected outcomes of this initiative are:

1. Development, demonstration and qualification of specific Intelligent Grid solutions required by utility company partners.

2. Design, implementation and validation of technologies, components and systems needed for the realization of Intelligent Grid.

3. Strategic alliance between BMPIC members with synergistic and complementary technologies for application to Intelligent Grid.

4. Commercial success for BMPIC members thru demonstration of their products for application to Intelligent Grid to local and international customers.

5. Establishing BCIT as a leader in research as well as manpower training for Intelligent Grid in Canada.

Author

Dr. Hassan Farhangi, Ph.D., M.Sc., and B.Sc., is the Director of the Group for Advanced Information Technology (GAIT) within the Technology Centre of British Columbia Institute of Technology in Burnaby, BC. Dr. Farhangi has more than 20 years of experience in academic and applied research. Before joining BCIT, he served as Chief Technical Officer (CTO) of a number of companies involved in the design and development of systems, components and solutions for the Intelligent Grid. Dr. Farhangi is a member of Association of Professional Engineers and Geoscientists of British Columbia (APEG), and a senior member of Institute of Electrical and Electronic Engineers (IEEE).

\section{Acknowledgments}

The author would like to thank the following BCIT organizations for their valuable contributions towards section $\mathrm{C}$ (Co-generation plants) of this paper:

Section C.1. (Boilers) by BCIT's School of Manufacturing and Industrial Process (MEIP)

Section C.2. (Photovoltaic Stations) by BCIT's School of Construction and the Environment (SoCE)

- Section C.3. (Micro-Wind Turbine) by BCIT's Technology Center

\section{References}

[1] S. J. Anders, "The emerging Smart Grid", Energy Policy Initiative Center, University of San Diego School of Law, Octo 2007, pp.4-8.

[2] P. Mazza, "Powering up the Smart Grid," Climate Solutions, July 2005, pp. $25-26$.

[3] J. Maire, "Profiling and Mapping of Intelligent Grid R\&D Programs," Electric Power Research Institute (EPRI) report, Dec 2006, pp. 124-126

[4] H. Farhangi, "Intelligent Micro Grid Research at BCIT," EnergyBiz Smart Grid supplement, July 2008., in press

[5] M. Tabatabaian, "Experimental investigation of power density enhancement for a small wind turbine augmented with a novel deflector" Renewable Energy, An International Journal, Elsevier, submitted (2007).

Notes

$\dagger \quad$ For more information on the members of BCIT's Microgrid Private Industry Consortium (BMPIC), please contact the author.

i† For more information on BCIT's WPD technology, please contact BCIT's Applied Resdearch Liasion Office (ARLO). 